\title{
Mechanism of Metal Chloride-Promoted Mukaiyama Aldol Reactions
}

\author{
Chiong Teck Wong and Ming Wah Wong* \\ Department of Chemistry, National University of Singapore, 3 Science Drive 3, Singapore \\ 117543 \\ chmwmw@nus.edu.sg
}

\section{Table of Contents ( 9 pages)}

Pages S2: Schematic energy diagram of the three reaction pathways, [2a], [2b] and [3], for the $\mathrm{TiCl}_{4}$-catalyzed reaction between trihydrosilyl enol ether and formaldehyde. The B3LYP/6-31G* reaction energies $(\Delta \mathrm{E})$ and barriers $\left(\Delta \mathrm{E}^{\neq}\right)$are in $\mathrm{kJ} \mathrm{mol}^{-1}$ (Figure S1).

Pages S3-S9: Cartesian coordinates and total energies and number of imaginary frequencies for B3LYP/6-31G* optimized geometries of equilibrium structures, transition states and products (Table S1). 


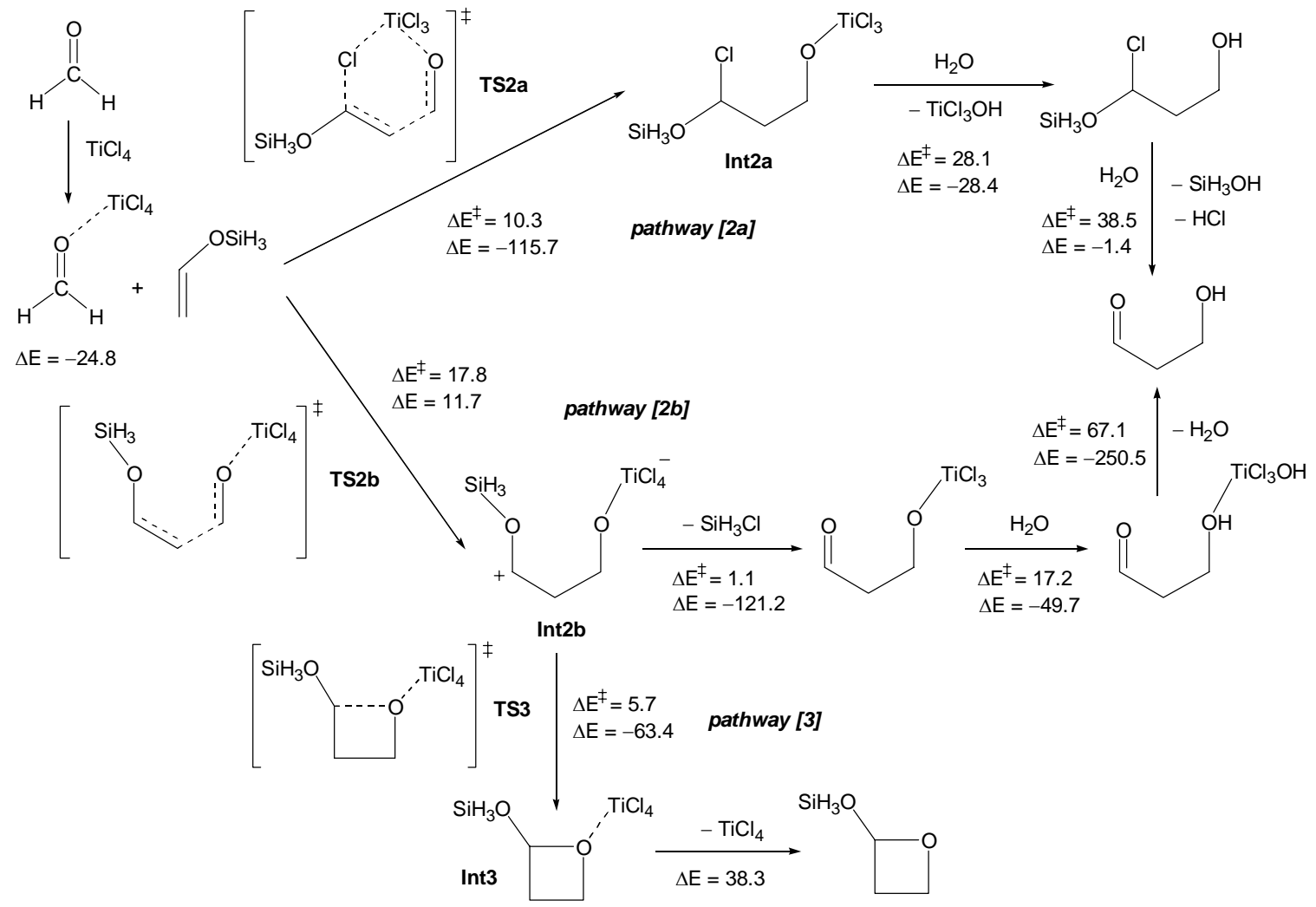

Figure S1. Schematic energy diagram of the three reaction pathways, [2a], [2b] and [3], for the $\mathrm{TiCl}_{4}$-catalyzed reaction between trihydrosilyl enol ether and formaldehyde. The B3LYP/6-31G* reaction energies $(\Delta \mathrm{E})$ and barriers $\left(\Delta \mathrm{E}^{\ddagger}\right)$ are in $\mathrm{kJ} \mathrm{mol}^{-1}$. 


\section{Table S1. Cartesian Coordinates (Á) of Optimized B3LYP/6-31G* Geometries of Reactants, Transition States, Intermediates and Products}

\begin{tabular}{|c|c|c|}
\hline \multicolumn{3}{|c|}{ trans-trihydrosilyl enol ether } \\
\hline \multicolumn{3}{|c|}{ Energy $=-444.53348$} \\
\hline \multicolumn{3}{|c|}{ No of imaginary frequency $=0$} \\
\hline $\mathrm{C}$ & 0.11137 & 2.35263 \\
\hline $\mathrm{H}$ & 0.15732 & 3.19379 \\
\hline $\mathrm{H}$ & 0.22202 & 2.53993 \\
\hline $\mathrm{C}$ & -0.08762 & 1.12499 \\
\hline $\mathrm{H}$ & -0.20768 & 0.92712 \\
\hline $\mathrm{O}$ & -0.19682 & 0.02886 \\
\hline $\mathrm{Si}$ & 0.06260 & -1.55031 \\
\hline $\mathrm{H}$ & 1.51415 & -1.86468 \\
\hline $\mathrm{H}$ & -0.55538 & -1.78317 \\
\hline $\mathrm{H}$ & -0.57487 & -2.40532 \\
\hline
\end{tabular}

\section{$\mathrm{CH}_{2}=\mathrm{O}$}

Energy $=-114.50047$

No of imaginary frequency $=0$

$\begin{array}{lll}\mathrm{O} & 0.67764 & 0.00000 \\ \mathrm{C} & -0.52896 & 0.00000 \\ \mathrm{H} & -1.12367 & 0.00000 \\ \mathrm{H} & -1.12367 & 0.00000\end{array}$

$\mathrm{AlCl}_{3}$

Energy $=-1623.23327$

No of imaginary frequency $=0$

$\begin{array}{llrr}\mathrm{Al} & 0.00000 & 0.00000 & 0.00000 \\ \mathrm{Cl} & 0.00000 & 0.00000 & 2.08657 \\ \mathrm{Cl} & 0.00000 & 1.80702 & -1.04328 \\ \mathrm{Cl} & 0.00000 & -1.80702 & -1.04328\end{array}$

TS

Energy $=-559.01608$

No of imaginary frequency $=1$

$\begin{array}{lrrr}\mathrm{O} & 0.71752 & -1.12878 & -0.21324 \\ \mathrm{C} & 0.63168 & -1.18557 & 1.05884 \\ \mathrm{H} & 1.56401 & -1.24323 & 1.63107 \\ \mathrm{H} & -0.22951 & -1.68589 & 1.52169 \\ \mathrm{C} & -0.07100 & 0.67120 & 1.74157 \\ \mathrm{H} & 0.31198 & 0.91313 & 2.72868 \\ \mathrm{H} & -1.09815 & 0.32399 & 1.70257 \\ \mathrm{C} & 0.42873 & 1.36532 & 0.65364 \\ \mathrm{H} & 1.28813 & 2.03430 & 0.75511 \\ \mathrm{O} & 0.05733 & 1.15134 & -0.56230 \\ \mathrm{Si} & -0.74815 & -0.29913 & -1.28485 \\ \mathrm{H} & -1.69396 & 0.61478 & -2.02021 \\ \mathrm{H} & -0.09172 & -0.99434 & -2.42044 \\ \mathrm{H} & -1.71194 & -1.06113 & -0.43058\end{array}$

cis-trihydrosilyl enol ether

Energy $=-444.53440$

No of imaginary frequency $=0$

$\begin{array}{rlrrr}-0.10217 & \mathrm{C} & 1.85042 & 0.55225 & 0.18350 \\ 0.57991 & \mathrm{C} & 1.22480 & -0.62128 & 0.08034 \\ -1.16520 & \mathrm{H} & 2.93003 & 0.57354 & 0.27802 \\ 0.37114 & \mathrm{H} & 1.33311 & 1.50750 & 0.17844 \\ 1.43792 & \mathrm{H} & 1.76679 & -1.56381 & 0.08807 \\ -0.44288 & \mathrm{O} & -0.11733 & -0.83603 & -0.04501 \\ 0.03995 & \mathrm{Si} & -1.32036 & 0.33064 & -0.10074 \\ 0.12815 & \mathrm{H} & -2.58323 & -0.42760 & -0.24186 \\ 1.37541 & \mathrm{H} & -1.34150 & 1.14177 & 1.14582 \\ -0.98627 & \mathrm{H} & -1.13275 & 1.24206 & -1.26108\end{array}$

$\mathrm{BCl}_{3}$

Energy $=-1405.56265$

No of imaginary frequency $=0$

$\begin{array}{llrr}\mathrm{B} & 0.00000 & 0.00000 & 0.00000 \\ \mathrm{Cl} & 0.00000 & 0.00000 & 1.75253 \\ \mathrm{Cl} & 0.00000 & 1.51774 & -0.87627 \\ \mathrm{Cl} & 0.00000 & -1.51774 & -0.87627\end{array}$

$\mathrm{GaCl}_{3}$

Energy $=-3303.68133$

No of imaginary frequency $=0$

$\begin{array}{lrrr}\mathrm{Ga} & 0.00000 & 0.00000 & 0.00000 \\ \mathrm{Cl} & 0.00000 & 0.00000 & 2.13225 \\ \mathrm{Cl} & 0.00000 & 1.84658 & -1.06613 \\ \mathrm{Cl} & 0.00000 & -1.84658 & -1.06612\end{array}$

$\mathrm{TiCl}_{4}$

Energy $=-2690.47849$

No of imaginary frequency $=0$

$\begin{array}{lrrr}\mathrm{Ti} & -0.00061 & 0.00022 & -0.00015 \\ \mathrm{Cl} & -0.52844 & 1.89403 & -0.93749 \\ \mathrm{Cl} & 2.11470 & -0.40326 & -0.32126 \\ \mathrm{Cl} & -1.18462 & -1.60246 & -0.87862 \\ \mathrm{Cl} & -0.40086 & 0.11140 & 2.13757\end{array}$

$\mathrm{H}_{2} \mathrm{O}$

Energy $=-76.40895$

No of imaginary frequency $=0$

$\begin{array}{llrr}\mathrm{O} & 0.00000 & 0.00000 & 0.11975 \\ \mathrm{H} & 0.00000 & 0.76157 & -0.47902 \\ \mathrm{H} & 0.00000 & -0.76157 & -0.47902\end{array}$


$\mathrm{H}_{2} \mathrm{C}=\mathrm{O} \cdots \mathrm{TiCl}_{4}$ complex

Energy $=-2804.99128$

No of imaginary frequency $=0$

$\begin{array}{lrr}\mathrm{O} & -0.56215 & 0.01170 \\ \mathrm{C} & 0.09613 & -0.00396 \\ \mathrm{H} & 1.19615 & -0.03190 \\ \mathrm{H} & -0.41029 & 0.01031 \\ \mathrm{Ti} & -0.02457 & 0.00067 \\ \mathrm{Cl} & -1.19945 & -1.86524 \\ \mathrm{Cl} & 0.41711 & -0.00662 \\ \mathrm{Cl} & -1.07098 & 1.94103 \\ \mathrm{Cl} & 2.06949 & -0.07288\end{array}$

$\mathrm{H}_{2} \mathrm{C}=\mathrm{O} \cdots \mathrm{AlCl}_{3}$ complex

Energy $=-1737.77598$

No of imaginary frequency $=0$

$\begin{array}{lrrr}\mathrm{O} & 0.00040 & 1.52684 & 0.84591 \\ \mathrm{C} & -0.01181 & 1.64769 & 2.06684 \\ \mathrm{H} & -0.02507 & 0.76833 & 2.72337 \\ \mathrm{H} & -0.00922 & 2.65553 & 2.49826 \\ \mathrm{Al} & 0.00150 & -0.13016 & -0.23484 \\ \mathrm{Cl} & -1.80226 & 0.10290 & -1.31309 \\ \mathrm{Cl} & -0.02530 & -1.58589 & 1.32926 \\ \mathrm{Cl} & 1.83241 & 0.08107 & -1.27129\end{array}$

Pre-TS $\pi-\pi$ complex $\left(\mathrm{TiCl}_{4}\right)$

Energy $=-3249.53258$

No of imaginary frequency $=0$

$\begin{array}{lrrr}\mathrm{O} & 0.79965 & 1.39497 & 0.91729 \\ \mathrm{C} & -0.11367 & 1.62030 & 1.70417 \\ \mathrm{H} & -0.20214 & 2.61394 & 2.15893 \\ \mathrm{H} & -0.80788 & 0.83407 & 2.02516 \\ \mathrm{H} & -4.21988 & -1.54114 & 1.75775 \\ \mathrm{H} & -3.52270 & -1.32915 & -0.58342 \\ \mathrm{Ti} & 1.43188 & -0.35616 & -0.32153 \\ \mathrm{H} & -5.66404 & -0.44061 & 0.14572 \\ \mathrm{C} & -2.39334 & 2.77369 & 0.42195 \\ \mathrm{C} & -2.74329 & 1.51526 & 0.12261 \\ \mathrm{H} & -1.78669 & 3.34108 & -0.27417 \\ \mathrm{H} & -2.76655 & 3.26686 & 1.31439 \\ \mathrm{H} & -2.40134 & 1.02993 & -0.79284 \\ \mathrm{O} & -3.50527 & 0.75552 & 0.94838 \\ \mathrm{Si} & -4.25707 & -0.69871 & 0.54256 \\ \mathrm{Cl} & 3.38455 & 0.06392 & 0.62492 \\ \mathrm{Cl} & -0.08383 & -1.48348 & 0.87576 \\ \mathrm{Cl} & 0.67121 & 0.95368 & -1.94996 \\ \mathrm{Cl} & 2.06428 & -2.05276 & -1.59139\end{array}$

Pre-TS $\pi-\pi$ complex $\left(\mathrm{AlCl}_{3}\right)$
$\mathrm{H}_{2} \mathrm{C}=\mathrm{O} \cdots \mathrm{BCl}_{3}$ complex

Energy $=-1520.07096$

No of imaginary frequency $=0$

$\begin{array}{rlrrr}-2.03605 & \mathrm{O} & -1.27712 & -0.81431 & 0.00016 \\ -3.06047 & \mathrm{C} & -1.26410 & -2.04302 & 0.00011 \\ -3.03781 & \mathrm{H} & -0.32021 & -2.59759 & 0.00001 \\ -4.03664 & \mathrm{H} & -2.22570 & -2.56439 & 0.00022 \\ 0.24750 & \mathrm{~B} & 0.06513 & 0.19259 & -0.00004 \\ 0.12444 & \mathrm{Cl} & -0.18609 & 1.11906 & -1.54011 \\ 2.40210 & \mathrm{Cl} & 1.54935 & -0.88648 & -0.00043 \\ 0.11146 & \mathrm{Cl} & -0.18551 & 1.11870 & 1.54042 \\ -0.50384 & & & & \end{array}$

$\mathrm{H}_{2} \mathrm{C}=\mathrm{O} \cdots \mathrm{GaCl}_{3}$ complex

Energy $=-3418.21897$

No of imaginary frequency $=0$

$\begin{array}{lrrr}\mathrm{O} & 1.62044 & -0.90247 & -0.00046 \\ \mathrm{C} & 1.70571 & -2.12325 & -0.00152 \\ \mathrm{H} & 0.80991 & -2.75812 & -0.00285 \\ \mathrm{H} & 2.70079 & -2.58666 & -0.00119 \\ \mathrm{Ga} & -0.13547 & 0.21029 & 0.00013 \\ \mathrm{Cl} & 0.12297 & 1.25907 & 1.86339 \\ \mathrm{Cl} & -1.57512 & -1.42414 & -0.00650 \\ \mathrm{Cl} & 0.12809 & 1.27008 & -1.85613\end{array}$

Pre-TS $\pi-\pi$ complex $\left(\mathrm{BCl}_{3}\right)$

Energy $=-1964.61513$

No of imaginary frequency $=0$

$\begin{array}{lrrr}\mathrm{O} & 0.33229 & -1.00180 & 0.32742 \\ \mathrm{C} & 1.55839 & -1.19746 & 0.12377 \\ \mathrm{H} & 1.82659 & -2.06992 & -0.46784 \\ \mathrm{H} & 2.3113 & -0.69859 & 0.73332 \\ \mathrm{~B} & -0.26624 & -0.01716 & 1.39334 \\ \mathrm{Cl} & -1.79241 & 0.64969 & 0.61448 \\ \mathrm{Cl} & 0.98279 & 1.31019 & 1.76033 \\ \mathrm{Cl} & -0.59823 & -1.08364 & 2.85575 \\ \mathrm{C} & 2.40303 & 0.31357 & -1.55224 \\ \mathrm{C} & 1.39917 & -0.06663 & -2.37937 \\ \mathrm{H} & 3.40082 & -0.08267 & -1.70817 \\ \mathrm{H} & 2.27000 & 1.15308 & -0.87799 \\ \mathrm{H} & 1.54610 & -0.84991 & -3.12873 \\ \mathrm{O} & 0.18177 & 0.45301 & -2.28190 \\ \mathrm{Si} & -1.25106 & -0.10279 & -3.01746 \\ \mathrm{H} & -2.17232 & 1.04676 & -3.01457 \\ \mathrm{H} & -0.88855 & -0.50490 & -4.40438 \\ \mathrm{H} & -1.79039 & -1.27154 & -2.29049\end{array}$

Pre-TS $\pi-\pi$ complex $\left(\mathrm{GaCl}_{3}\right)$ 
Energy $=-2182.32225$

No of imaginary frequency $=0$

$\begin{array}{lrrr}\mathrm{O} & -0.98544 & -0.67565 & -0.32626 \\ \mathrm{C} & -2.12930 & -0.33747 & 0.06218 \\ \mathrm{H} & -2.78561 & -1.10722 & 0.47110 \\ \mathrm{H} & -2.56408 & 0.62085 & -0.23102 \\ \mathrm{Al} & 0.25844 & 0.32355 & -1.35156 \\ \mathrm{Cl} & 2.16132 & -0.13653 & -0.50372 \\ \mathrm{Cl} & -0.40845 & 2.34348 & -1.04587 \\ \mathrm{Cl} & -0.05253 & -0.41238 & -3.32488 \\ \mathrm{C} & -1.97769 & 0.81842 & 2.18072 \\ \mathrm{C} & -1.24319 & -0.21692 & 2.65282 \\ \mathrm{H} & -3.02034 & 0.90532 & 2.46722 \\ \mathrm{H} & -1.49675 & 1.66201 & 1.69613 \\ \mathrm{H} & -1.70283 & -1.02509 & 3.22832 \\ \mathrm{O} & 0.05427 & -0.33174 & 2.38908 \\ \mathrm{Si} & 1.06442 & -1.66180 & 2.73947 \\ \mathrm{H} & 2.39834 & -1.10209 & 3.02085 \\ \mathrm{H} & 0.48332 & -2.31449 & 3.94482 \\ \mathrm{H} & 1.07109 & -2.61312 & 1.60955\end{array}$

\section{TS2a $\left(\mathrm{TiCl}_{4}\right)$}

Energy $=-3862.76244$

No of imaginary frequency $=1$

$\begin{array}{lrrr}\mathrm{O} & 1.60855 & 0.47049 & 0.02132 \\ \mathrm{C} & 2.45008 & -0.14903 & -0.70842 \\ \mathrm{H} & 2.39383 & -1.24075 & -0.77130 \\ \mathrm{H} & 3.45290 & 0.28483 & -0.77768 \\ \mathrm{C} & 2.18338 & 0.24989 & -2.68997 \\ \mathrm{H} & 3.02520 & -0.32153 & -3.07008 \\ \mathrm{H} & 2.29358 & 1.32915 & -2.64128 \\ \mathrm{C} & 0.92467 & -0.25995 & -2.92703 \\ \mathrm{H} & 0.77899 & -1.31475 & -3.17067 \\ \mathrm{O} & -0.14851 & 0.47441 & -2.78696 \\ \mathrm{Si} & -1.79490 & -0.02701 & -2.91783 \\ \mathrm{H} & -1.77886 & -1.44895 & -3.33455 \\ \mathrm{H} & -2.44428 & 0.20030 & -1.61824 \\ \mathrm{H} & -2.35977 & 0.83542 & -3.97851 \\ \mathrm{Ti} & -0.00378 & 0.05297 & 1.16051 \\ \mathrm{Cl} & -0.80270 & 2.05286 & 0.56300 \\ \mathrm{Cl} & -1.85669 & -0.56410 & 2.27471 \\ \mathrm{Cl} & 1.35083 & -0.00513 & 2.92161 \\ \mathrm{Cl} & -0.17256 & -1.81984 & -0.18529\end{array}$

TS2a $\left(\mathrm{BCl}_{3}\right)$

Energy $=-1964.61613$

No of imaginary frequency $=1$

$\begin{array}{llrl}\mathrm{O} & 0.91258 & -0.94594 & -0.81720 \\ \mathrm{C} & 2.07391 & -0.56307 & -0.49445 \\ \mathrm{H} & 2.37534 & 0.47043 & -0.65502\end{array}$

Energy $=-3862.76490$

No of imaginary frequency $=0$

\begin{tabular}{lrrr}
\multicolumn{4}{l}{ No of imaginary frequency $=0$} \\
$\mathrm{O}$ & -1.01602 & -0.81306 & -0.14749 \\
$\mathrm{C}$ & -2.16406 & -0.47047 & 0.18291 \\
$\mathrm{H}$ & -2.82992 & -1.21919 & 0.61913 \\
$\mathrm{H}$ & -2.57384 & 0.50511 & -0.09224 \\
$\mathrm{Ga}$ & 0.27925 & 0.31317 & -1.19404 \\
$\mathrm{Cl}$ & 2.20228 & -0.16011 & -0.31691 \\
$\mathrm{Cl}$ & -0.49633 & 2.32473 & -0.82981 \\
$\mathrm{Cl}$ & -0.06891 & -0.44565 & -3.19276 \\
$\mathrm{C}$ & -2.06156 & 0.78819 & 2.43920 \\
$\mathrm{C}$ & -1.32906 & -0.25653 & 2.87461 \\
$\mathrm{H}$ & -3.10247 & 0.87088 & 2.73268 \\
$\mathrm{H}$ & -1.59586 & 1.62027 & 1.92115 \\
$\mathrm{H}$ & -1.77790 & -1.06307 & 3.46006 \\
$\mathrm{O}$ & -0.02754 & -0.38056 & 2.58803 \\
$\mathrm{Si}$ & 0.95884 & -1.72817 & 2.90087 \\
$\mathrm{H}$ & 2.31418 & -1.20386 & 3.15092 \\
$\mathrm{H}$ & 0.40538 & -2.39128 & 4.11427 \\
$\mathrm{H}$ & 0.92655 & -2.67344 & 1.76391
\end{tabular}

\section{TS2b $\left(\mathrm{TiCl}_{4}\right)$}

Energy $=-3862.76166$

No of imaginary frequency $=1$

$\begin{array}{lrrr}\mathrm{O} & 0.72659 & 0.82058 & 0.43847 \\ \mathrm{C} & 1.91187 & 0.91293 & 0.94500 \\ \mathrm{H} & 2.12639 & 1.82952 & 1.50615 \\ \mathrm{H} & 2.75632 & 0.55307 & 0.34252 \\ \mathrm{C} & 2.19143 & -0.43088 & 2.27345 \\ \mathrm{H} & 3.20031 & -0.23812 & 2.62988 \\ \mathrm{H} & 2.05887 & -1.29822 & 1.63189 \\ \mathrm{C} & 1.15398 & -0.10549 & 3.13516 \\ \mathrm{H} & 1.30324 & 0.59554 & 3.96339 \\ \mathrm{O} & -0.05847 & -0.49515 & 2.88592 \\ \mathrm{Si} & -1.53719 & 0.11739 & 3.55276 \\ \mathrm{H} & -1.90707 & 1.35615 & 2.84675 \\ \mathrm{H} & -2.50217 & -0.98240 & 3.40438 \\ \mathrm{H} & -1.22136 & 0.39699 & 4.97797 \\ \mathrm{Ti} & -0.10621 & 0.04781 & -1.21454 \\ \mathrm{Cl} & 1.64627 & -1.41599 & -1.18701 \\ \mathrm{Cl} & -0.98022 & -0.94918 & -3.03224 \\ \mathrm{Cl} & 0.21432 & 2.02286 & -2.20527 \\ \mathrm{Cl} & -1.98894 & -0.23244 & 0.01048\end{array}$

TS2b $\left(\mathrm{BCl}_{3}\right)$

Energy $=-1964.61504$

No of imaginary frequency $=1$

$\begin{array}{lllr}\mathrm{O} & 0.35845 & -0.96078 & 0.30335 \\ \mathrm{C} & 1.60143 & -1.14618 & 0.09599 \\ \mathrm{H} & 1.86869 & -2.06761 & -0.41709\end{array}$




$\begin{array}{lrrrlrrr}\mathrm{H} & 2.82716 & -1.34142 & -0.40099 & \mathrm{H} & 2.33803 & -0.67839 & 0.74918 \\ \mathrm{~B} & -0.20912 & -0.03434 & -1.37030 & \mathrm{~B} & -0.23083 & 0.01076 & 1.34715 \\ \mathrm{Cl} & -0.09996 & -0.19295 & -3.19868 & \mathrm{Cl} & -1.82242 & 0.60167 & 0.61691 \\ \mathrm{Cl} & 0.09206 & 1.72770 & -0.80935 & \mathrm{Cl} & 0.96408 & 1.41668 & 1.63767 \\ \mathrm{Cl} & -1.77293 & -0.71194 & -0.67096 & \mathrm{Cl} & -0.47417 & -0.99885 & 2.87245 \\ \mathrm{C} & 2.44135 & -0.35334 & 1.78729 & \mathrm{C} & 2.34906 & 0.20481 & -1.47963 \\ \mathrm{C} & 1.25186 & 0.23863 & 2.08387 & \mathrm{C} & 1.35151 & -0.14947 & -2.34170 \\ \mathrm{H} & 3.32392 & 0.26616 & 1.67166 & \mathrm{H} & 3.34922 & -0.18130 & -1.64867 \\ \mathrm{H} & 2.57385 & -1.41252 & 1.98242 & \mathrm{H} & 2.22860 & 1.08334 & -0.85400 \\ \mathrm{H} & 1.12421 & 1.31939 & 2.00746 & \mathrm{H} & 1.49296 & -0.94589 & -3.07895 \\ \mathrm{O} & 0.18298 & -0.47593 & 2.39092 & \mathrm{O} & 0.15189 & 0.38074 & -2.24788 \\ \mathrm{Si} & -1.35984 & 0.11172 & 2.85218 & \mathrm{Si} & -1.31126 & -0.17640 & -2.94775 \\ \mathrm{H} & -2.30673 & -0.97779 & 2.56537 & \mathrm{H} & -2.20410 & 0.99373 & -2.96952 \\ \mathrm{H} & -1.28832 & 0.36765 & 4.31407 & \mathrm{H} & -0.95647 & -0.62000 & -4.32347 \\ \mathrm{H} & -1.63923 & 1.36959 & 2.12885 & \mathrm{H} & -1.84721 & -1.31420 & -2.17583\end{array}$

TS2a $\left(\mathrm{AlCl}_{3}\right)$

Energy $=-2182.32175$

No of imaginary frequency $=1$

$\begin{array}{lrr}\mathrm{O} & 1.20008 & -1.00165 \\ \mathrm{C} & 2.30040 & -0.56906 \\ \mathrm{H} & 2.53949 & 0.49476 \\ \mathrm{H} & 3.12735 & -1.27722 \\ \mathrm{Al} & -0.20847 & -0.01125 \\ \mathrm{Cl} & 0.06866 & -0.13343 \\ \mathrm{Cl} & 0.15521 & 1.96113 \\ \mathrm{Cl} & -2.00432 & -0.89877 \\ \mathrm{C} & 2.38576 & -0.58743 \\ \mathrm{C} & 1.24294 & 0.11619 \\ \mathrm{H} & 3.33998 & -0.07737 \\ \mathrm{H} & 2.36953 & -1.66858 \\ \mathrm{H} & 1.25153 & 1.20670 \\ \mathrm{O} & 0.07386 & -0.48822 \\ \mathrm{Si} & -1.43001 & 0.19739 \\ \mathrm{H} & -2.39573 & -0.90673 \\ \mathrm{H} & -1.25910 & 0.61237 \\ \mathrm{H} & -1.74122 & 1.36757\end{array}$

TS2a $\left(\mathrm{GalCl}_{3}\right)$

Energy $=-3862.76244$

No of imaginary frequency $=1$

$\begin{array}{lrr}\mathrm{O} & 1.30614 & -1.05812 \\ \mathrm{C} & 2.37449 & -0.56083 \\ \mathrm{H} & 2.56897 & 0.51098 \\ \mathrm{H} & 3.25509 & -1.20975 \\ \mathrm{Ga} & -0.16484 & -0.00636 \\ \mathrm{Cl} & 0.08254 & -0.07741 \\ \mathrm{Cl} & 0.21285 & 1.99274 \\ \mathrm{Cl} & -1.98563 & -0.94918 \\ \mathrm{C} & 2.36273 & -0.64790\end{array}$

$\begin{array}{ll}-0.61740 & \mathrm{O} \\ -0.16309 & \mathrm{C} \\ -0.22157 & \mathrm{H} \\ -0.09239 & \mathrm{H} \\ -1.34459 & \mathrm{Al} \\ -3.44986 & \mathrm{Cl} \\ -0.52995 & \mathrm{Cl} \\ -0.60367 & \mathrm{Cl} \\ 2.06635 & \mathrm{C} \\ 2.31529 & \mathrm{C} \\ 2.14574 & \mathrm{H} \\ 2.15843 & \mathrm{H} \\ 2.37013 & \mathrm{H} \\ 2.40326 & \mathrm{O} \\ 2.88131 & \mathrm{~S} \\ 2.76620 & \mathrm{H} \\ 4.29788 & \mathrm{H} \\ 2.03807 & \mathrm{H}\end{array}$

TS2b $\left(\mathrm{AlCl}_{3}\right)$

Energy $=-2182.32186$

No of imaginary frequency $=1$

$\begin{array}{lrrr}\mathrm{O} & 0.52650 & -1.08940 & 0.07909 \\ \mathrm{C} & 1.75994 & -1.23976 & -0.23106 \\ \mathrm{H} & 2.02359 & -2.15169 & -0.77104 \\ \mathrm{H} & 2.53491 & -0.82259 & 0.41840 \\ \mathrm{Al} & -0.22620 & 0.04606 & 1.34052 \\ \mathrm{Cl} & -1.99107 & 0.83372 & 0.40611 \\ \mathrm{Cl} & 1.31454 & 1.52270 & 1.66331 \\ \mathrm{Cl} & -0.61549 & -1.17788 & 3.04300 \\ \mathrm{C} & 2.33936 & 0.14092 & -1.68280 \\ \mathrm{C} & 1.31421 & -0.16691 & -2.54166 \\ \mathrm{H} & 3.34149 & -0.18893 & -1.93952 \\ \mathrm{H} & 2.23940 & 1.00419 & -1.03113 \\ \mathrm{H} & 1.43709 & -0.91421 & -3.33186 \\ \mathrm{O} & 0.11470 & 0.32151 & -2.35652 \\ \mathrm{Si} & -1.36684 & -0.20201 & -3.06534 \\ \mathrm{H} & -1.99227 & -1.22444 & -2.20875 \\ \mathrm{H} & -2.17754 & 1.01583 & -3.22991 \\ \mathrm{H} & -0.97677 & -0.78655 & -4.37682\end{array}$

TS2b $\left(\mathrm{GalCl}_{3}\right)$

Energy $=-3862.76166$

No of imaginary frequency $=1$

$\begin{array}{rlrrr}-0.42168 & \mathrm{O} & 0.57297 & -1.14832 & -0.17956 \\ 0.08145 & \mathrm{C} & 1.84209 & -1.21256 & -0.49748 \\ -0.00858 & \mathrm{H} & 2.13528 & -2.14230 & -0.99798 \\ 0.07715 & \mathrm{H} & 2.57744 & -0.87964 & 0.24590 \\ -1.13380 & \mathrm{Ga} & -0.17873 & 0.02820 & 1.14452 \\ -3.28458 & \mathrm{Cl} & -1.95590 & 0.88706 & 0.17817 \\ -0.25698 & \mathrm{Cl} & 1.41307 & 1.51653 & 1.50345 \\ -0.38994 & \mathrm{Cl} & -0.64455 & -1.20873 & 2.86920 \\ 2.11946 & \mathrm{C} & 2.31407 & 0.07751 & -1.73273\end{array}$




$\begin{array}{lrrrlrrr}\mathrm{C} & 1.23950 & 0.08533 & 2.43493 & \mathrm{C} & 1.31667 & -0.14281 & -2.68354 \\ \mathrm{H} & 3.32810 & -0.18963 & 2.31329 & \mathrm{H} & 3.32996 & -0.16973 & -2.03388 \\ \mathrm{H} & 2.29609 & -1.72848 & 2.20336 & \mathrm{H} & 2.20426 & 0.96529 & -1.11228 \\ \mathrm{H} & 1.29010 & 1.16793 & 2.56880 & \mathrm{H} & 1.46126 & -0.83715 & -3.51871 \\ \mathrm{O} & 0.06001 & -0.47957 & 2.46948 & \mathrm{O} & 0.12830 & 0.31813 & -2.48210 \\ \mathrm{Si} & -1.44792 & 0.22246 & 2.95021 & \mathrm{Si} & -1.37827 & -0.20257 & -3.17944 \\ \mathrm{H} & -2.39405 & -0.90335 & 2.93344 & \mathrm{H} & -1.98070 & -1.22291 & -2.30916 \\ \mathrm{H} & -1.21668 & 0.71054 & 4.33451 & \mathrm{H} & -2.17192 & 1.02570 & -3.33911 \\ \mathrm{H} & -1.80204 & 1.34188 & 2.06091 & \mathrm{H} & -0.98051 & -0.78148 & -4.49057\end{array}$

\section{Int2a}

Energy $=-3249.57755$

No of imaginary frequency $=0$

$\begin{array}{lrrr}\mathrm{O} & 0.96197 & -0.04081 & -0.47296 \\ \mathrm{C} & 1.86494 & -0.11310 & 0.61739 \\ \mathrm{H} & 2.87635 & -0.06142 & 0.19946 \\ \mathrm{H} & 1.73234 & -1.08465 & 1.10302 \\ \mathrm{C} & 1.66129 & 1.02328 & 1.61953 \\ \mathrm{H} & 1.54120 & 1.97589 & 1.09522 \\ \mathrm{H} & 2.57899 & 1.08936 & 2.21946 \\ \mathrm{C} & 0.52451 & 0.83294 & 2.62064 \\ \mathrm{H} & 0.51564 & 1.65279 & 3.34632 \\ \mathrm{O} & 0.64741 & -0.39537 & 3.23833 \\ \mathrm{Si} & -0.28690 & -0.91424 & 4.54648 \\ \mathrm{H} & 0.51634 & -1.98622 & 5.17582 \\ \mathrm{H} & -0.47647 & 0.22484 & 5.48458 \\ \mathrm{H} & -1.59991 & -1.44079 & 4.10989 \\ \mathrm{Ti} & -0.02820 & -0.12523 & -1.87562 \\ \mathrm{Cl} & -1.13162 & 1.00500 & 1.79664 \\ \mathrm{Cl} & -1.57149 & -1.67921 & -1.60903 \\ \mathrm{Cl} & 1.27483 & -0.68682 & -3.56922 \\ \mathrm{Cl} & -0.93802 & 1.84427 & -2.28834\end{array}$

TS3

Energy $=-3249.53124$

No of imaginary frequency $=1$

$\begin{array}{lrrr}\mathrm{H} & 2.54843 & -0.81282 & 0.42532 \\ \mathrm{C} & 2.45108 & 0.26255 & 0.25409 \\ \mathrm{C} & 2.24391 & 1.20940 & 1.38521 \\ \mathrm{O} & 0.37123 & -0.21860 & 1.46387 \\ \mathrm{C} & 1.17626 & 0.57073 & 2.30219 \\ \mathrm{H} & 1.63831 & -0.07814 & 3.05594 \\ \mathrm{H} & 0.58112 & 1.33974 & 2.80935 \\ \mathrm{O} & 2.54596 & 0.70459 & -0.91761 \\ \mathrm{H} & 3.20654 & 1.36076 & 1.89913 \\ \mathrm{Si} & 2.70343 & -0.18447 & -2.47367 \\ \mathrm{H} & 1.91988 & 2.17063 & 0.97631 \\ \mathrm{H} & 3.12299 & -1.56063 & -2.16210 \\ \mathrm{H} & 1.45756 & 0.04756 & -3.20704 \\ \mathrm{H} & 3.83451 & 0.59771 & -3.02442\end{array}$

\section{Int2b}

Energy $=-3249.52643$

No of imaginary frequency $=0$

$\begin{array}{lrrr}\mathrm{O} & 0.15790 & 1.05952 & -0.94080 \\ \mathrm{C} & -0.33285 & 2.33715 & -0.66153 \\ \mathrm{H} & -0.38888 & 2.91660 & -1.59299 \\ \mathrm{H} & 0.30665 & 2.87822 & 0.04719 \\ \mathrm{C} & -1.75642 & 2.16001 & -0.05915 \\ \mathrm{H} & -2.26175 & 3.13635 & -0.00555 \\ \mathrm{H} & -1.70201 & 1.71695 & 0.93853 \\ \mathrm{C} & -2.50195 & 1.30472 & -1.00831 \\ \mathrm{H} & -2.60758 & 1.65608 & -2.04097 \\ \mathrm{O} & -3.02496 & 0.19517 & -0.78773 \\ \mathrm{Si} & -3.23340 & -0.89982 & 0.64790 \\ \mathrm{H} & -4.53079 & -0.29757 & 1.06213 \\ \mathrm{H} & -3.47243 & -2.23341 & 0.09129 \\ \mathrm{H} & -2.22392 & -0.66656 & 1.68622 \\ \mathrm{Ti} & 1.13271 & -0.17155 & -0.03687 \\ \mathrm{Cl} & -0.66582 & -1.77251 & -0.58078 \\ \mathrm{Cl} & 2.57981 & -1.45368 & -1.14999 \\ \mathrm{Cl} & 0.56545 & -0.42077 & 2.14950 \\ \mathrm{Cl} & 2.68012 & 1.43615 & 0.50813\end{array}$

\section{Int3}

Energy $=-3249.55719$

No of imaginary frequency $=0$

$\begin{array}{lrrr}\mathrm{H} & 2.63955 & -0.63642 & 0.42865 \\ \mathrm{C} & 2.28223 & 0.39635 & 0.37487 \\ \mathrm{C} & 2.67985 & 1.35748 & 1.51008 \\ \mathrm{O} & 0.87347 & 0.41612 & 0.87104 \\ \mathrm{C} & 1.19498 & 1.42773 & 1.88712 \\ \mathrm{H} & 0.91409 & 1.06572 & 2.87653 \\ \mathrm{H} & 0.68333 & 2.36669 & 1.66183 \\ \mathrm{O} & 2.41526 & 0.93808 & -0.87066 \\ \mathrm{H} & 3.32456 & 0.90886 & 2.26847 \\ \mathrm{Si} & 2.65057 & 0.14926 & -2.34694 \\ \mathrm{H} & 3.11784 & 2.28436 & 1.13830 \\ \mathrm{H} & 3.25171 & -1.18367 & -2.08586 \\ \mathrm{H} & 1.38006 & 0.02469 & -3.09368 \\ \mathrm{H} & 3.59746 & 1.00511 & -3.09914\end{array}$




$\begin{array}{lrrrrrrr}\mathrm{Ti} & -1.05484 & -0.17325 & 0.35482 & \mathrm{Ti} & -1.07513 & -0.32754 & 0.19379 \\ \mathrm{Cl} & 0.29372 & -1.71750 & -1.03847 & \mathrm{Cl} & 0.21418 & -1.94964 & -0.66758 \\ \mathrm{Cl} & -2.06035 & 1.35211 & 1.74144 & \mathrm{Cl} & -1.58018 & -0.28600 & 2.36011 \\ \mathrm{Cl} & -2.66006 & -1.70867 & 0.53134 & \mathrm{Cl} & -2.99081 & -1.19440 & -0.50897 \\ \mathrm{Cl} & -0.95660 & 1.32026 & -1.34996 & \mathrm{Cl} & -1.26767 & 1.62756 & -0.83866\end{array}$

\begin{tabular}{|c|c|c|c|c|c|c|c|}
\hline \multicolumn{4}{|c|}{$\mathrm{TiCl}_{3} \mathrm{CH}_{2} \mathrm{CH}_{2} \mathrm{CHO}$} & \multicolumn{4}{|c|}{ oxetane } \\
\hline \multicolumn{4}{|c|}{ Energy $=-2498.03842$} & \multicolumn{4}{|c|}{ Energy $=-559.06260$} \\
\hline \multicolumn{4}{|c|}{ No of imaginary frequency $=0$} & \multicolumn{4}{|c|}{ No of imaginary frequency $=0$} \\
\hline $\mathrm{O}$ & 0.92249 & -0.21303 & -0.56537 & $\mathrm{H}$ & 1.17590 & -1.04464 & 1.32511 \\
\hline $\mathrm{C}$ & 1.75069 & -0.34289 & -1.70796 & $\mathrm{C}$ & 0.56908 & -0.31542 & 0.77475 \\
\hline $\mathrm{H}$ & 1.91879 & -1.41433 & -1.87424 & $\mathrm{C}$ & 0.26807 & 1.00982 & 1.50803 \\
\hline $\mathrm{H}$ & 2.71305 & 0.12788 & -1.48130 & $\mathrm{O}$ & -0.82469 & -0.68296 & 0.78018 \\
\hline $\mathrm{C}$ & 1.09562 & 0.31064 & -2.92417 & $\mathrm{C}$ & -1.18945 & 0.51525 & 1.52050 \\
\hline $\mathrm{H}$ & 1.80546 & 0.23366 & -3.76201 & $\mathrm{H}$ & -1.59388 & 0.26063 & 2.50614 \\
\hline $\mathrm{H}$ & 0.89011 & 1.36941 & -2.74183 & $\mathrm{H}$ & -1.91992 & 1.11936 & 0.97140 \\
\hline $\mathrm{C}$ & -0.18962 & -0.38754 & -3.33281 & $\mathrm{O}$ & 1.09370 & -0.21613 & -0.51213 \\
\hline $\mathrm{H}$ & -0.10256 & -1.49233 & -3.45389 & $\mathrm{H}$ & 0.74521 & 1.12827 & 2.48373 \\
\hline $\mathrm{O}$ & -1.23826 & 0.17856 & -3.52945 & $\mathrm{Si}$ & 0.10831 & -0.20507 & -1.87180 \\
\hline $\mathrm{Ti}$ & -0.01882 & -0.00479 & 0.86474 & $\mathrm{H}$ & 0.45970 & 1.89128 & 0.89271 \\
\hline $\mathrm{Cl}$ & -1.49250 & -1.63797 & 1.00570 & $\mathrm{H}$ & -0.54327 & -1.51167 & -2.13877 \\
\hline $\mathrm{Cl}$ & -1.03474 & 1.94764 & 0.79425 & $\mathrm{H}$ & -0.92811 & 0.86383 & -1.82133 \\
\hline $\mathrm{Cl}$ & 1.33755 & -0.06993 & 2.60223 & $\mathrm{H}$ & 1.04970 & 0.09877 & -2.97781 \\
\hline
\end{tabular}

\section{$\mathrm{TiCl}_{3} \mathrm{OH}$}

Energy $=-2306.09496$

No of imaginary frequency $=0$

$\begin{array}{lrrr}\mathrm{Ti} & -0.03367 & 0.01764 & 0.24247 \\ \mathrm{Cl} & -0.54632 & 1.89688 & -0.77586 \\ \mathrm{Cl} & 2.10682 & -0.42401 & 0.02128 \\ \mathrm{Cl} & -1.25102 & -1.59677 & -0.61959 \\ \mathrm{O} & -0.41824 & 0.17108 & 1.93866 \\ \mathrm{H} & -1.17480 & 0.34957 & 2.51729\end{array}$

$\mathrm{SiH}_{3} \mathrm{Cl}$-catalyzed aldol TS

Energy $=-1310.52710$

No of imaginary frequency $=1$

$\begin{array}{lrrr}\mathrm{O} & 0.62249 & -1.45992 & 0.32750 \\ \mathrm{C} & 0.62581 & -1.46300 & 1.60467 \\ \mathrm{H} & 1.60382 & -1.46227 & 2.08773 \\ \mathrm{H} & -0.19046 & -1.96152 & 2.13968 \\ \mathrm{C} & -0.02328 & 0.37822 & 2.37420 \\ \mathrm{H} & 0.10099 & 0.13991 & 3.42636 \\ \mathrm{H} & -1.03966 & 0.37965 & 1.99190 \\ \mathrm{C} & 0.94944 & 1.15691 & 1.78595 \\ \mathrm{H} & 1.93178 & 1.23810 & 2.25595 \\ \mathrm{O} & 0.90332 & 1.73915 & 0.62186 \\ \mathrm{Si} & -0.42885 & 2.32346 & -0.32470 \\ \mathrm{H} & -0.80124 & 3.56948 & 0.40099 \\ \mathrm{H} & 0.13779 & 2.63982 & -1.64001\end{array}$

\section{$\mathrm{SiH}_{3} \mathrm{OH}$}

Energy $=-367.14285$

No of imaginary frequency $=0$

$\begin{array}{lrrr}\mathrm{Si} & -0.53014 & 0.04495 & -0.05475 \\ \mathrm{H} & -1.21758 & -0.16972 & 1.25193 \\ \mathrm{H} & -1.00553 & -0.96174 & -1.03189 \\ \mathrm{H} & -0.89958 & 1.41003 & -0.53030 \\ \mathrm{O} & 1.11960 & -0.16642 & 0.05226 \\ \mathrm{H} & 1.58790 & 0.42344 & 0.65866\end{array}$

$\mathrm{CH}_{2}=\mathrm{O} \cdots \mathrm{SiH}_{3} \mathrm{Cl}$

Energy $=-866.03627$

No of imaginary frequency $=0$

\begin{tabular}{lrrr}
$\mathrm{O}$ & -2.04675 & -0.72320 & 0.00303 \\
$\mathrm{C}$ & -2.58955 & 0.35749 & 0.00292 \\
$\mathrm{H}$ & -3.69457 & 0.45369 & 0.00383 \\
$\mathrm{H}$ & -2.01661 & 1.30396 & 0.00191 \\
$\mathrm{Si}$ & 1.21182 & -0.94305 & -0.00049 \\
$\mathrm{Cl}$ & 0.98693 & 1.13456 & -0.00236 \\
$\mathrm{H}$ & 0.60510 & -1.49538 & 1.22875 \\
$\mathrm{H}$ & 2.67234 & -1.20838 & -0.00226 \\
$\mathrm{H}$ & 0.60177 & -1.49793 & -1.22693 \\
& & \\
$\mathrm{HCl}$ & & \\
$\mathrm{H}$ Energy = -460.79569 \\
\multicolumn{4}{l}{ No of imaginary frequency $=0$}
\end{tabular}




$\begin{array}{lrrrrrrr}\mathrm{H} & -1.57290 & 1.39629 & -0.30086 & \mathrm{Cl} & 0.00000 & 0.00000 & 0.07166 \\ \mathrm{Si} & -0.89926 & -1.91942 & -0.66969 & \mathrm{H} & 0.00000 & 0.00000 & -1.21826 \\ \mathrm{Cl} & 0.06404 & -0.43557 & -2.16646 & & & & \\ \mathrm{H} & -0.73442 & -3.03615 & -1.63024 & & & & \\ \mathrm{H} & -2.14128 & -1.09377 & -0.73278 & & & & \\ \mathrm{H} & -1.30785 & -2.72787 & 0.56896 & & & & \end{array}$

